\title{
A Simple and Novel Noninvasive Method of Estimating Markedly Elevated Pulmonary Vascular Resistance in Patients with Pre-capillary Pulmonary Hypertension
}

\section{Ya-Nan Zhai}

China-Japan Friendship Hospital

Ai-Li Li (D echoaili@163.com )

China-Japan Friendship Hospital

Xin-Cao Tao

China-Japan Friendship Hospital

Wan-Mu Xie

China-Japan Friendship Hospital

Qian Gao

China-Japan Friendship Hospital

\section{Yu Zhang}

China-Japan Friendship Hospital

\section{Ai-Hong Chen}

China-Japan Friendship Hospital

Jie-Ping Lei

China-Japan Friendship Hospital

Min Liu

China-Japan Friendship Hospital

Zhen-Guo Zhai

China-Japan Friendship Hospital

Jin-Gang Zheng

China-Japan Friendship Hospital

\section{Research Article}

Keywords: Pulmonary hypertension, Echocardiography, Pulmonary vascular resistance

Posted Date: July 13th, 2021

DOI: https://doi.org/10.21203/rs.3.rs-660018/v1 
License: (c) (i) This work is licensed under a Creative Commons Attribution 4.0 International License. Read Full License 


\section{Abstract}

Background: Several echocardiographic methods to estimate pulmonary vascular resistance (PVR) in patients with pulmonary hypertension $(\mathrm{PH})$ have been proposed. So far, most studies have focused on relatively low PVR. We aimed to clarify the clinical usefulness of our new echocardiographic index of evaluating markedly elevated PVR in pre-capillary PH patients.

Methods: We studied 129 consecutive patients with pre-capillary $\mathrm{PH}$. We estimated the mean pulmonary artery pressure using echocardiography $\left(\mathrm{mPAP}_{\text {Echo }}\right)$ and measured LV internal diameter at end diastole

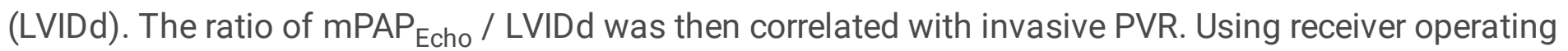
characteristic curve analysis, a cutoff value for the index was generated to identify patients with PVR > 15 Wood units (WU).

Results: $\mathrm{mPAP}_{\text {Echo }} /$ LVIDd correlated well with PVR $(r=0.70, \mathrm{P}<0.0001)$. There was a better correlation between PVR and $\mathrm{mPAP}_{E_{\text {Echo }}}$ / LVIDd in patients with PVR $>15 \mathrm{WU}$ compared with $\mathrm{TRV}^{2} / \mathrm{TVI}_{\mathrm{RVOT}}$ and SPAP $_{\text {Echo }} /$ LVIDd. A cut-off value of 1.14 had an $80.0 \%$ sensitivity and $74.7 \%$ specificity to determine PVR $>15 \mathrm{WU}(\mathrm{AUC}=0.840, p<0.0001)$.

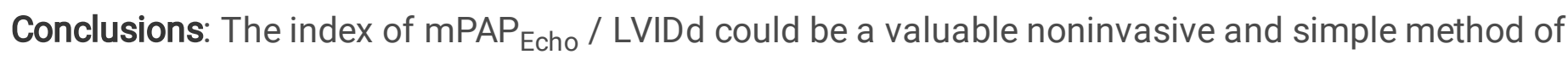
estimating markedly elevated PVR in pre-capillary $\mathrm{PH}$ patients.

\section{Background}

Pulmonary vascular resistance (PVR), calculated as the transpulmonary gradient divided by pulmonary blood flow, is the important parameter of hemodynamic evaluation in the management of pulmonary hypertension (PH). Assessment of PVR usually requires right heart catheterization (RHC), which is invasive and difficult to popularize and continuously monitor patients.

Several echocardiographic methods to estimate PVR have been proposed previously. Abbas et al. in 2003 found a good correlation $(r=0.93)$ between the invasively measured PVR and the ratio of the tricuspid regurgitation velocity (TRV) to the velocity time integral of the right ventricular outflow tract (TVI $\mathrm{RVOT})$ [1]. In 2013 Abbas et al. demonstrated a more robust association between PVR and $\mathrm{TRV}^{2} / \mathrm{TVI}_{\mathrm{RVOT}}$, including patients with a PVR $>6 \mathrm{WU}$ [2]. However, we noticed that their study population consisted of patients with various cardiac and pulmonary pathologies and relatively low PVR $(80 \% \leq 6 \mathrm{WU})$, and do not take right atrial pressure into account.

It has been reported that echocardiographic methods are useful for estimating the mean pulmonary artery pressure (PAP) [3]. And it is consistent with the formula being used for the RHC. PVR increased in patients with reduced pulmonary blood flow, and left ventricular volume can reflect pulmonary blood flow. Studies have confirmed that left ventricular end-diastolic volume (LVEDV) is reduced in idiopathic pulmonary arterial hypertension (IPAH) in proportion to reduced pulmonary flow [4-5]. Therefore, we use 
estimating mPAP (mPAP Echo ) and LV internal diameter at end diastole (LVIDd) surrogate for transpulmonary pressure and pulmonary blood flow respectively. Our hospital admits patients with various types of $\mathrm{PH}$, with a wide range of PVR, especially patients with high PVR. A large proportion of patients with high PVR are chronic thromboembolic pulmonary hypertension patients who evaluate the risk of pulmonary endarterectomy. Measurement of patient's haemodynamic status including PVR is crucial. We hypothesized that we could establish a simpler and more accurate noninvasive method to estimate PVR.

The main objective of our study was to investigate whether the new simple method $\mathrm{mPAP}_{\text {Echo }} /$ LVIDd would provide better estimation of PVR in situations of markedly elevated PVR.

\section{Methods}

\section{Study Population}

A total of 430 consecutive patients referred for transthoracic echocardiography and RHC evaluation of known or suspected PH between June 2015 and December 2020 were included. A subsequent of 163 patients were excluded due to an interval over 3 days between $\mathrm{RHC}$ and echocardiography. Patients with congenital heart disease were not included. Of the remaining 258 patients, 224 were diagnosed with $\mathrm{PH}$. Pre-capillary PH was defined as pulmonary arterial pressure (mPAP) of $\geq 25 \mathrm{mmHg}$ at rest, pulmonary arterial wedge pressure $\leq 15 \mathrm{mmHg}$ and pulmonary vascular resistance (PVR) $>3$ Wood units [6]. 11 patients with PAWP $>15 \mathrm{mmHg}$ and 84 patients without pulmonary regurgitation were excluded for a final study cohort of 129 pre-capillary PH patients (Figure 1). Table 1 described the diagnosis of all the participants according to 2015 ESC Guidelines [7]. This study was approved by China-Japan Friendship Hospital's Human Research Ethics Committee.

\section{Right Heart Catheterization}

A 7F Swan-Ganz catheter Philips Allura X-PER FD20 flat-plate angiography system (Baxter Inc) was used to measure systolic, diastolic, and mean pulmonary arterial pressure (PAP), mean right atrial pressure (RAP), and mean pulmonary capillary wedge pressure (PCWP). Cardiac output was measured using the Fick method, which calculated the cardiac index $(\mathrm{Cl})$. The transpulmonary gradient (TPG) was calculated by subtracting the mean PAP from the PCWP. Pulmonary vascular resistance (PVR) (Wood units, WU) was calculated by dividing the TPG by the cardiac output.

\section{Echocardiography}

Within 3 days of RHC, two-dimensional and Doppler echocardiography were performed using the Vivid E95 ultrasound system (General Electric Healthcare, Vingmed, Horten, Norway) with a M5S transducer. The subjects were placed in the left lateral position, and at least three consecutive beats were stored. 
Two-dimensional (2D) and Doppler echocardiography were performed in accordance with current guidelines [8]. Analysis of the images was performed offline using the EchoPac software version 201 (General Electric Healthcare, Vingmed, Horten, Norway). LV internal diameter at end-diastole (LVIDd) was acquired in the parasternal long-axis view by M-mode. The ultrasound beam is aligned so that it is perpendicular to the interventricular septum and posterior wall at a level of the mitral leaflet tips (Figure 2). $\mathrm{TVI}_{\mathrm{RVOT}}$ was obtained by placing a $1-\mathrm{mm}$ to $2-\mathrm{mm}$ pulsed wave Doppler sample volume in the proximal right ventricular outflow tract when imaged from the parasternal short-axis view. The average of

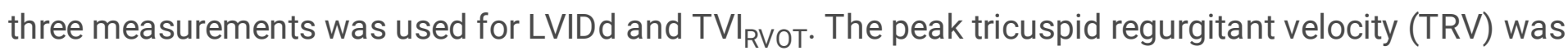
measured as the highest of the velocities obtained from the lower parasternal and apical multiple views. The early diastolic pulmonary regurgitation velocity (PRV) was measured as the highest of the velocities obtained from the parasternal short-axis view (Figure 2).

Noninvasive estimation of RAP was based on the size and collapse index of the IVC and was scored as either $3 \mathrm{mmHg}, 8 \mathrm{mmHg}$, or $15 \mathrm{mmHg}$ [8]. Doppler echocardiographic determination of mean PAP $\left(\mathrm{mPAP}_{\text {echo }}\right)$ was estimated as $4 \times(\text { early PRV })^{2}+$ estimated RAP. Doppler echocardiographic determination of systolic PAP ( SPAP $\left._{\text {echo }}\right)$ was estimated as $4 \times \mathrm{TRV}^{2}+$ estimated RAP.

The first method was based on the index of $\mathrm{mPAP}_{\mathrm{Echo}} / \mathrm{LVIDd}$. The second method was based on the ratio of $\mathrm{SPAP}_{\text {Echo }} / \mathrm{LVIDd}$. The TRV2/TVI $\mathrm{RVOT}$ ratio was also calculated, as described by Abbas et al ${ }^{[2]}$.

\section{Statistical Analysis}

Continuous data are presented as the mean \pm standard deviation, and categorical data are presented as percentages. Correlations between variables were assessed using Pearson's correlation coefficient. Receiver operating characteristics (ROC) curve were performed to determine echocardiographic cutoff values for detecting PVR > $6 \mathrm{WU}$ and $15 \mathrm{WU}$. Intra-observer and inter-observer reproducibility were assessed in randomly selected 30 subjects. Inter-observer reproducibility was tested by two independent observers. Inter-observer and intra-observer reproducibility were evaluated by means of intraclass correlation coefficient (ICC). Statistical analyses were performed using SPSS 17.0 software (SPSS Inc, Chicago, USA).

\section{Results}

The main clinical, hemodynamic and basic echocardiographic characteristics of the studied patients are presented in Table 1.

Table 1

Baseline Characteristics of Study Population 


$\begin{array}{ll}\text { Variable } & \text { Pre-capilla } \\ \text { Demographic characteristics } & \\ \text { Age (years) } & 48 \pm 14 \\ \text { Male }(\%) & 52(40 \%) \\ \text { BSA }\left(\mathrm{m}^{2}\right) & 1.7 \pm 0.8 \\ \text { Aetiology of PH } & \\ \text { PAH } & 51 \\ \text { CTEPH } & 69 \\ \text { Lung disease } & 3 \\ \text { Others } & 6\end{array}$

WHO functional class

I/II

61

III/IV

68

Elevated BNP or NT-proBNP (\%)

$80(80 \%)$

Right heart caheterization

Systolic PAP (mmHg)

$81 \pm 24$

Mean PAP $(\mathrm{mmHg})$

$46 \pm 14$

Mean RAP $(\mathrm{mmHg})$

$3.3 \pm 5.1$

PAWP $(\mathrm{mmHg})$

$7.1 \pm 5.0$

PVR (WU)

$14.6 \pm 7.5$

$\mathrm{Cl}\left(\mathrm{L} / \mathrm{min} / \mathrm{m}^{2}\right)$

$2.1 \pm 0.9$

Echocardiography

LVIDd (mm)

$39.3 \pm 5.4$

LVEF (\%)

$69 \pm 6$

RA minor-axis dimension ( $\mathrm{mm}$ )

$50.9 \pm 9.4$

RV basal diameter ( $\mathrm{mm}$ )

$47.4 \pm 7.0$

$\mathrm{RV} / \mathrm{LV}$ basal diameter ratio

$1.4 \pm 0.3$

HR (beats/min)

$78 \pm 15$

$\operatorname{TRV}(\mathrm{m} / \mathrm{s})$ $430 \pm 67$ 


\begin{tabular}{|c|c|}
\hline TRPG (mmHg) & $75.9 \pm 22.5$ \\
\hline PREDG $(\mathrm{mmHg})$ & $34.2 \pm 12.3$ \\
\hline $\mathrm{TVI}_{\mathrm{RVOT}}(\mathrm{cm})$ & $11.1 \pm 3.8$ \\
\hline $\mathrm{TRV}^{2} / \mathrm{TVI}_{\mathrm{RVOT}}$ & $8.6 \pm 3.7$ \\
\hline $\mathrm{mPAP}_{\text {Echo }} /$ LVIDd & $\begin{array}{l}1.1 \pm 0.4 \\
2.2 \pm 0.8\end{array}$ \\
\hline $\mathrm{sPAP}_{\mathrm{Echo}} / \mathrm{LVIDd}$ & $15.6 \pm 3.5$ \\
\hline TAPSE (mm) & $10.0 \pm 2.4$ \\
\hline $\mathrm{S}^{\prime}(\mathrm{cm} / \mathrm{s})$ & $28.8 \pm 8.3$ \\
\hline \multicolumn{2}{|l|}{ RV FAC (\%) } \\
\hline $\begin{array}{l}\text { BSA, body surface } \\
\text { pulmonary hyperter } \\
\text { proBNP, N-terminal } \\
\text { right atrial pressure } \\
\text { WU, wood units; Cl, } \\
\text { ejection fraction; RA } \\
\text { velocity; TRPG, tric } \\
\text { diastolic gradient; I } \\
\text { tract; mPAP Echo, ech } \\
\text { determination of sy } \\
\text { ventricular fraction }\end{array}$ & $\begin{array}{l}\text { nsion; CTEPH, chronic thromboembolic } \\
\text { ease induced PAH; BNP, brain natriuretic; NT- } \\
\text { tide; PAP, pulmonary artery pressure; RAP, } \\
\text { sure; PVR, pulmonary vascular resistance; } \\
\text { end-diastolic diameter; LVEF, left ventricular } \\
\text { heart rate; TRV, tricuspid regurgitation } \\
\text { PREDG, pulmonary regurgitation early- } \\
\text { ty time integral of the right ventricular outflow } \\
\text { an PAP; sPAP } \text { Echo, echocardiographic } \\
\text { olane systolic excursion; RVFAC, right }\end{array}$ \\
\hline
\end{tabular}

Linear regression analysis between $\mathrm{PVR}$ and $\mathrm{TRV}^{2} / \mathrm{TVI}_{\mathrm{RVOT}}$ revealed a weak correlation $(r=0.47, \mathrm{P}<0.001$;

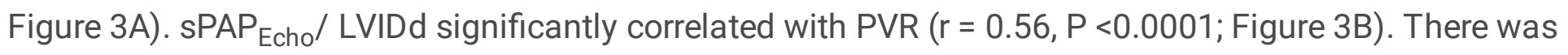
a better correlation between PVR and $\mathrm{mPAP}_{\text {Echo }} / \operatorname{LVIDd}(r=0.70, \mathrm{P}<0.0001$; Figure $3 \mathrm{C})$

For patients with $\mathrm{PVR} \leq 15 \mathrm{WU}$, there were similar correlations with both $\mathrm{TRV}^{2} / \mathrm{TVI}_{\mathrm{RVOT}}$ and $\mathrm{mPAP} \mathrm{Echo}_{\mathrm{O}}$ /

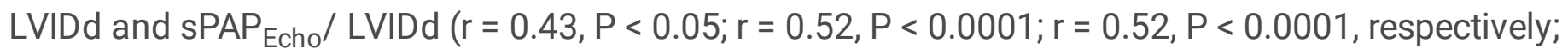
Figures 3D F). However, in patients with PVR $>15 \mathrm{WU}$, the correlation markedly improved with $\mathrm{mPAP}_{\text {Echo }} / \mathrm{LVIDd}$ compared with $\mathrm{TRV}^{2} / \mathrm{TVI}_{\mathrm{RVO}}$ and sPAP $\mathrm{Echo} / \mathrm{LVIDd}(\mathrm{r}=0.60, \mathrm{P}\langle 0.0001 ; \mathrm{r}=0.21, \mathrm{P}\rangle$ $0.05 ; r=0.37, P<0.05$, respectively; Figures $3 G \sim 1)$.

In the ROC curve analysis, the $\mathrm{TRV}^{2} / \mathrm{TVI}_{\mathrm{RVOT}} \geq 5.18$ had an $87.2 \%$ sensitivity and $100.0 \%$ specificity to determine PVR $>6 \mathrm{WU}$. Its area under the curve (AUC) is $0.942(\mathrm{P}<0.01,95 \% \mathrm{Cl}, 0.86-1.00)$, which is

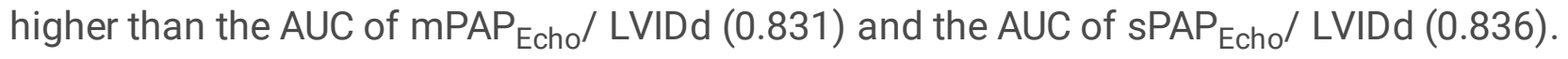

As shown in Figure 4 , the $\mathrm{mPAP}_{\text {Echo }} / \mathrm{LVIDd} \geq 1.14$ had an $80.0 \%$ sensitivity and $74.7 \%$ specificity to determine PVR $>15$ WU. Its AUC is $0.840(P<0.0001,95 \% \mathrm{Cl}, 0.76-0.92)$, which is higher than the AUC of 
$\mathrm{TRV}^{2} / \mathrm{TVI}_{\mathrm{RVOT}}(0.772)$ and the AUC of $\mathrm{SPAP}_{\text {Echo }} / \operatorname{LVIDd}(0.827)$.

\section{Reproducibility}

The inter-observer ICC was 0.83 for $\mathrm{TVI}_{\mathrm{RVOT}}$ and 0.97 for LVIDd, and the intra-observer ICC was 0.88 and 0.99 for $\mathrm{TVI}_{\mathrm{RVOT}}$ and LVIDd. LVIDd demonstrated better inter-observer and intra-observer reproducibility than $\mathrm{TVI}_{\mathrm{RVOT}}$.

\section{Discussion}

This study demonstrates that the index of $\mathrm{mPAP}_{\mathrm{Echo}} /$ LVIDd represents a simpler and better method of estimating markedly elevated PVR in pre-capillary $\mathrm{PH}$ patients.

A number of echocardiographic methods have been proposed for the noninvasive estimation of PVR [1$2,9-12]$. However, most methods acquired formulas containing various empirical constants, which would affect the accuracy of the estimating PVR results. The guidelines of the American Society of Echocardiography recommended the noninvasive estimation of PVR should not be used as a substitute for invasive evaluation of PVR[3]. However, pulmonary vascular resistance still needs to be evaluated in $\mathrm{PH}$ patients, especially continuous preoperative and postoperative monitoring in IPAH and CTEPH patients. PVR higher than $1200 \mathrm{dyn} \cdot \mathrm{s} \cdot \mathrm{cm}^{-5}(15 \mathrm{WU})$ is considered to be severe and is correlated with an increased risk of post-operative mortality [13-14]. We proposed a simple method to help clinically identify patients with extremely high PVR values (>15 WU) instead of generating a regression equation compared to previously reported methods.

Our results showed that both Abbas et al [2] and our new methods correlate similarly for patients with lower PVR values (PVR $\leq 15 \mathrm{WU})$. However, for PVR $>15 \mathrm{WU}$, the correlation appeared stronger with $\mathrm{mPAP}_{\text {Echo }}$ / LVIDd compared with $\mathrm{TRV}^{2} / \mathrm{TVI}_{\mathrm{RVOT}}$. Moreover, the AUC of $\mathrm{mPAP}_{\text {Echo }}$ / LVIDd is higher than the AUC of $\mathrm{TRV}^{2} / \mathrm{TVI}_{\mathrm{RVOT}}$ to determine PVR $>15 \mathrm{WU}$. The new method has its own advantages. The underlying explanations are as following:

The echocardiographic estimation of mean PAP is more consistent with PVR formula. The mean PAP reflects the driving pressure that is required for pulmonary blood flow more accurately than systolic PAP. PR is reported to occur in almost $75 \%$ of the population [15]. Dilation of the annulus and main pulmonary artery are usually accompanied by $\mathrm{PH}$ patients. PR secondary to $\mathrm{PH}$ is common, thus it is easy to obtain the complete PR Doppler signal. It has been reported that the early-diastolic PA-RV pressure gradients derived from the peak early-diastolic PR velocity is useful for estimating the mean PA pressure [3, 16-17]. In our study, TR could be obtained in 117 of 129 patients (90\%). Among these patients, 10 had severe TR, 41 had not well-defined TR signal quality, which may lead to overestimation or underestimation of pulmonary arterial pressure [18-20]. Abbas et al. method [1-2] and Haddad et al. method using TRV to calculate systolic PAP and Lindquist et al. method ${ }^{[9]}$ using TRV to calculate mean PAP may be inaccurate 
in patients under these circumstances. Extremely severe PR is rare in patients with $\mathrm{PH}$. Therefore, we consider that the mean PAP estimated by PR Doppler signal is more reliable when PR is present. TRV can be measured to estimate systolic PAP when PR is absent.

Compared to the method of Abbas et al [2] and Kaga et al [10], the new method also takes into account the contribution of RAP to estimate mPAP. Current guidelines have suggested that RAP predicts prognosis of PAH [7]. For patients with PVR $>15$ WU, RAP will also be higher. So, the estimated RAP will contribute more to $m P A P_{E c h o}$ in patients with PVR $>15$ WU. Although Haddad et al. [12] took RAP into account, according to the size and collapse index of the IVC, RAP was estimated to be $10 \mathrm{mmHg}, 15 \mathrm{mmHg}$, or $20 \mathrm{mmHg}$, which would lead to overestimation of RAP.

$\mathrm{PH}$ results in the reduced of pulmonary blood flow, which also causes the reduced of the blood flow back to the left atrial and ventricular. In addition, there is a leftward displacement of the interventricular septum during diastole of LV in sever $\mathrm{PH}$ patients, due to the presence of right ventricular pressure overload [2122], which will further lead to the compression of the LV cavity. Grzegorz et al. [5] reported that LVEDV in IPAH patients correlated inversely with PVR and LVEDV is reduced in $\mathrm{PH}$ patients in proportion to reduced pulmonary flow. Therefore, LVEDV represents pulmonary blood flow in pre-capillary $\mathrm{PH}$ and is significantly reduced in patients with extremely high PVR. LVIDd, as an alternative indicator of LVEDV, is easier to obtain and more suitable for routine applications. Moreover, LVIDd had better repeatability and accuracy than $\mathrm{TVI}_{\mathrm{ROVT}}$. In patients with $\mathrm{PH}$, the dilatation of main pulmonary artery caused pulmonary valve to move forward. When obtaining the flow velocity in the RV outflow tract, the Doppler sampling line had a larger angle with the direction of blood flow, which would affect the accuracy of the measurement of $\mathrm{TVI}_{\mathrm{ROVT}}$. In addition, when the velocity of the RV outflow tract was low, the contour of the spectrum was not clearly displayed, which would affect the repeatability of the measurement.

Our novel index has utility for predicting patients with markedly elevated PVR, which is of great value in etiological diagnosis, disease severity and continuous monitoring of PVR before and after treatment.

\section{Limitations}

There are several limitations in this study. Although the clinical condition of the patients was stable during each examination, $\mathrm{RHC}$ and echocardiography were not performed simultaneously, which affected the accuracy of the comparison. Second, this study was only applicable to patients with pre-capillary PH. However, we usually pay more attention to the PVR of pre-capillary PH patients rather than post-capillary $\mathrm{PH}$ patients in clinical practice. Third, this study was a single-center retrospective study. Further prospective multicenter studies involving larger patient populations are required to confirm the results.

\section{Conclusion}

The novel, simple and noninvasive index, $\mathrm{mPAP}_{\text {Echo }}$ / LVIDd, can be clinically used in the routine echocardiographic evaluation for $\mathrm{PH}$ and can be a valuable noninvasive and simple method of 
estimating markedly elevated PVR in pre-capillary $\mathrm{PH}$ patients. As compared with conventional methods, the ratio of $\mathrm{mPAP}_{\text {Echo }} / \mathrm{LVIDd}>1.14$ showed good sensitivity and specificity for identifying markedly elevated PVR (>15 WU).

\section{Declarations}

\section{Acknowledgements}

The author would like to thank the Pulmonary Vascular Disease Team and the Cardiology Team of ChinaJapan Friendship Hospital for their support of this study.

\section{Author contributions}

Ai-Li Li created the idea and designed the study. Ai-Li Li and Ya-Nan Zhai performed research, collected data, analyzed the results and wrote the manuscript. Xin-Cao Tao provided the data of RHC. Wan-Mu X

ie, Qian Gao helped in collecting the cases. Yu Zhang, Ai-Hong Chen helped organizing data. Jie-Ping Lei helped analyzed the statistical data. Min Liu, Zhen-Guo Zhai and Jin-Gang Zheng gave support of the study. All authors have given approval to the final version of the manuscript.

\section{Funding}

This study is supported by Capital's Funds for Health Improvement and Research (2020-2-4063), National key clinical specialties construction program (2020-QTL-009), the National Natural Science Foundation of China (81871328), the Beijing Natural Science Foundation (7182149).

\section{Availability of data and materials}

The datasets used and analyzed during the current study are available from the corresponding author on reasonable request.

\section{Ethics approval and consent to participate}

The study protocol was approved by China-Japan Friendship Hospital's Human Research Ethics Committee (2020-95-K59). All patients gave informed written consent before inclusion.

\section{Consent for publication}

Not applicable 


\section{Competing interests}

The authors declare that they have no competing interests

\section{Author details}

${ }^{1}$ Department of Cardiology, China-Japan Friendship Hospital, Beijing, China. ${ }^{2}$ Institute of Respiratory Medicine, Chinese Academy of Medical Sciences, Beijing, China. ${ }^{3}$ National Clinical Research Center for Respiratory Diseases, Beijing, China. ${ }^{4}$ Department of Pulmonary and Critical Care Medicine, China-Japan Friendship Hospital, Beijing, China. ${ }^{5}$ Data and Project Management Unit, Institute of Clinical Medical Sciences, China-Japan Friendship Hospital, Beijing, China. ${ }^{6}$ Department of Radiology, China-Japan Friendship Hospital, Beijing, China.

\section{References}

1. Abbas AE, Fortuin FD, Schiller NB, Appleton CP, Moreno CA, Lester SJ. A simple method for noninvasive estimation of pulmonary vascular resistance. J Am Coll Cardiol. 2003;41(6):1021-7.

2. Abbas AE, Franey LM, Marwick T, Maeder MT, Kaye DM, Vlahos AP, et al. Noninvasive assessment of pulmonary vascular resistance by Doppler echocardiography. J Am Soc Echocardiogr. 2013;26(10):1170-1177.

3. Rudski LG, Lai WW, Afilalo J, Hua L, Handschumacher MD, Chandrasekaran K, et al. Guidelines for the echocardiographic assessment of the right heart in adults: a report from the American Society of Echocardiography endorsed by the European Association of Echocardiography, a registered branch of the European Society of Cardiology, and the Canadian Society of Echocardiography. J Am Soc Echocardiogr.2010; 23(7):685-713.

4. vanWolferen SA, Marcus JT, Boonstra A, Marques KM, Bronzwaer JG, Spreeuwenberg MD, et al. Prognostic value of right ventricular mass, volume, and function in idiopathic pulmonary arterial hypertension. Eur Heart J 2007;28(10):1250-7.

5. Kopeć G, Moertl D, Miszalski-Jamka T, Waligóra M, Tyrka A, Sarnecka A, et al. Left ventricular mass is preserved in patients with idiopathic pulmonary arterial hypertension and Eisenmenger's syndrome. Heart Lung Circ.2014;23(5):454-61.

6. Simonneau G, Montani D, Celermajer DS, Denton CP, Gatzoulis MA, Krowka M, Williams PG, Souza R. Haemodynamic definitions and updated clinical classification of pulmonary hypertension. Eur Respir J.2019;53(1):1801-1913.

7. Galiè N, Humbert M, Vachiery JL, Gibbs S, Lang I, Torbicki A, et al. 2015 ESC/ERS Guidelines for the diagnosis and treatment of pulmonary hypertension: The Joint Task Force for the Diagnosis and Treatment of Pulmonary Hypertension of the European Society of Cardiology (ESC) and the European Respiratory Society (ERS): Endorsed by: Association for European Paediatric and 
Congenital Cardiology (AEPC), International Society for Heart and Lung Transplantation (ISHLT). Eur Heart J. 2016;37(1):67-119.

8. Lang RM, Badano LP, Mor-Avi V, Afilalo J, Armstrong A, Ernande L, et al. Recommendations for cardiac chamber quantification by echocardiography in adults: an update from the American Society of Echocardiography and the European Association of Cardiovascular Imaging. J Am Soc Echocardiogr. 2015;28(1):1-39.e14.

9. Lindqvist P, Söderberg S, Gonzalez MC, Tossavainen E, Henein MY. Echocardiography based estimation of pulmonary vascular resistance in patients with pulmonary hypertension: a simultaneous Doppler echocardiography and cardiac catheterization study. Eur J Echocardiogr. 2011;12(12):961-6.

10. Kaga S, Mikami T, Murayama M, Okada K, Masauzi N, Nakabachi M, et al. A new method to estimate pulmonary vascular resistance using diastolic pulmonary artery-right ventricular pressure gradients derived from continuous-wave Doppler velocity measurements of pulmonary regurgitation. Int $\mathrm{J}$ Cardiovasc Imaging. 2017 Jan;33(1):31-38.

11. Haddad F, Zamanian R, Beraud AS, Schnittger I, Feinstein J, Peterson T, et al. A novel non-invasive method of estimating pulmonary vascular resistance in patients with pulmonary arterial hypertension. J Am Soc Echocardiogr. 2009;22(5):523-9.

12. Scapellato F, Temporelli PL, Eleuteri E, Corra U, Imparato A, Giannuzzi P. Accurate noninvasive estimation of pulmonary vascular resistance by Doppler echocardiography in patients with chronic failure heart failure. J Am Coll Cardiol 2001;37(7):1813-9.

13. Jenkins D. Pulmonary endarterectomy: the potentially curative treatment for patients with chronic thromboembolic pulmonary hypertension. Eur Respir Rev. 2015;24(136):263-71.

14. Jenkins D, Madani M, Fadel E, D'Armini AM, Mayer E. Pulmonary endarterectomy in the management of chronic thromboembolic pulmonary hypertension. Eur Respir Rev 2017;26(143):160111.

15. Choong CY, Abascal VM, Weyman J, Levine RA, Gentile F, Thomas JD, et al. Prevalence of valvular regurgitation by Doppler echocardiography in patients with structurally normal hearts by twodimensional echocardiography. Am Heart J. 1989;117(3):636-42.

16. Milan A, Magnino C, Veglio F. Echocardiographic indexes for the non-invasive evaluation of pulmonary hemodynamics. J Am Soc Echocardiogr. 2010;23(3):225-39.

17. Abbas AE, Fortuin FD, Schiller NB, Appleton CP, Moreno CA, Lester SJ. Echocardiographic determination of mean pulmonary artery pressure. Am J Cardiol 2003;92(11):1373-6.

18. Fei B, Fan T, Zhao L, Pei X, Shu X, Fang X, et al. Impact of severe tricuspid regurgitation on accuracy of systolic pulmonary arterial pressure measured by Doppler echocardiography: Analysis in an unselected patient population. Echocardiography. 2017;347(7):1082-1088.

19. Amsallem M, Sternbach JM, Adigopula S, Kobayashi Y, Vu TA, Zamanian R, et al. Addressing the Controversy of Estimating Pulmonary Arterial Pressure by Echocardiography. J Am Soc Echocardiogr. 2016;29(2):93-102. 
20. Cordina RL, Playford D, Lang I, Celermajer DS. State-of-the-Art Review: Echocardiography in Pulmonary Hypertension. Heart Lung Circ. 2019;28(9):1351-1364.

21. Tonelli AR, Plana JC, Heresi GA, Dweik RA. Prevalence and prognostic value of left ventricular diastolic dysfunction in idiopathic and heritable pulmonary arterial hypertension. Chest. 2012;141(6):1457-1465.

22. Marcus JT, Gan CT, Zwanenburg JJ, Boonstra A, Allaart CP, Go“ tte MJ, et al. Interventricular mechanical asynchrony in pulmonary arterial hypertension: left-to-right delay in peak shortening is related to right ventricular overload and left ventricular underfilling. J Am Coll Cardiol. 2008;51(7):750-7.

\section{Figures}




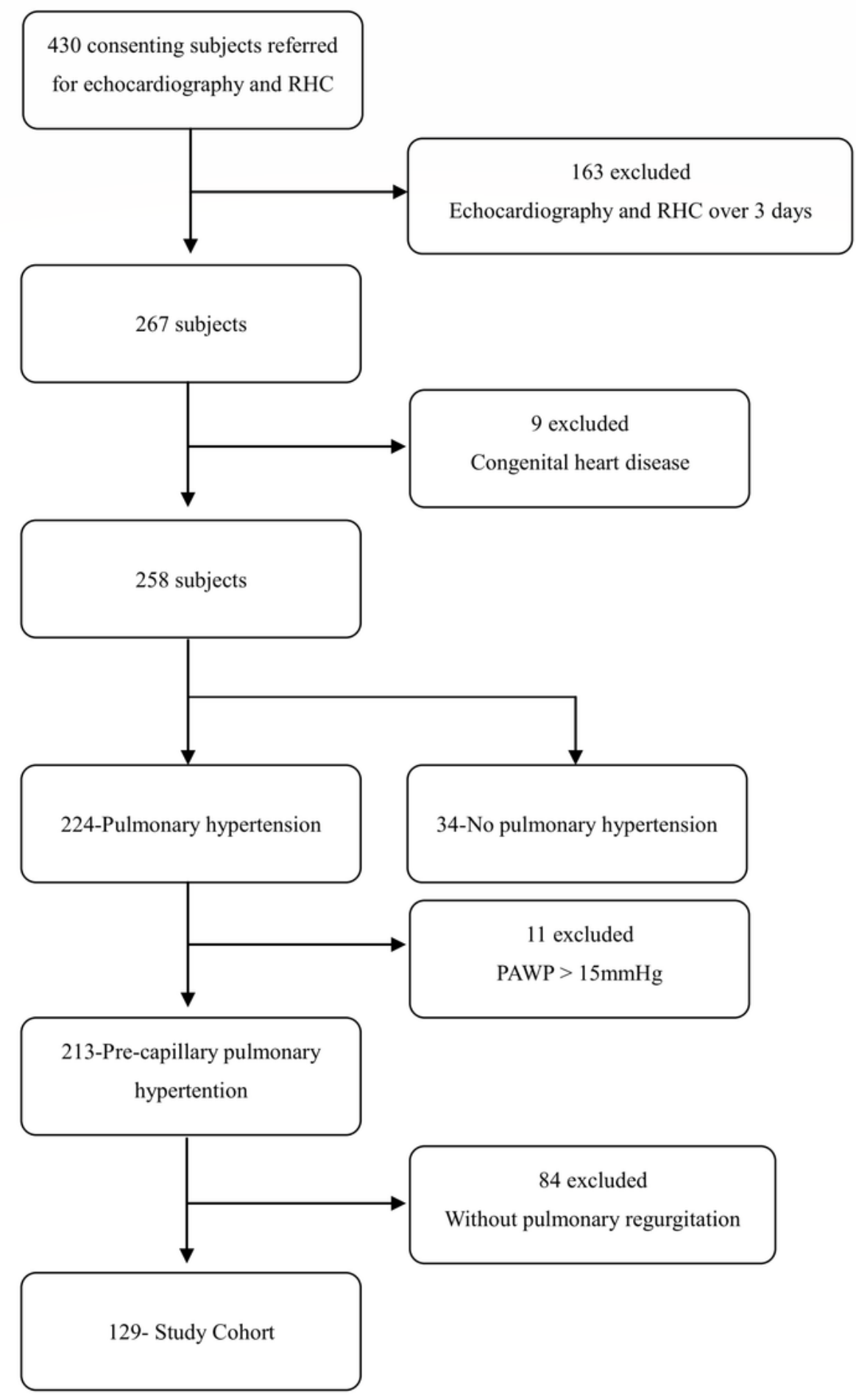

Figure 1

Study flow diagram. 


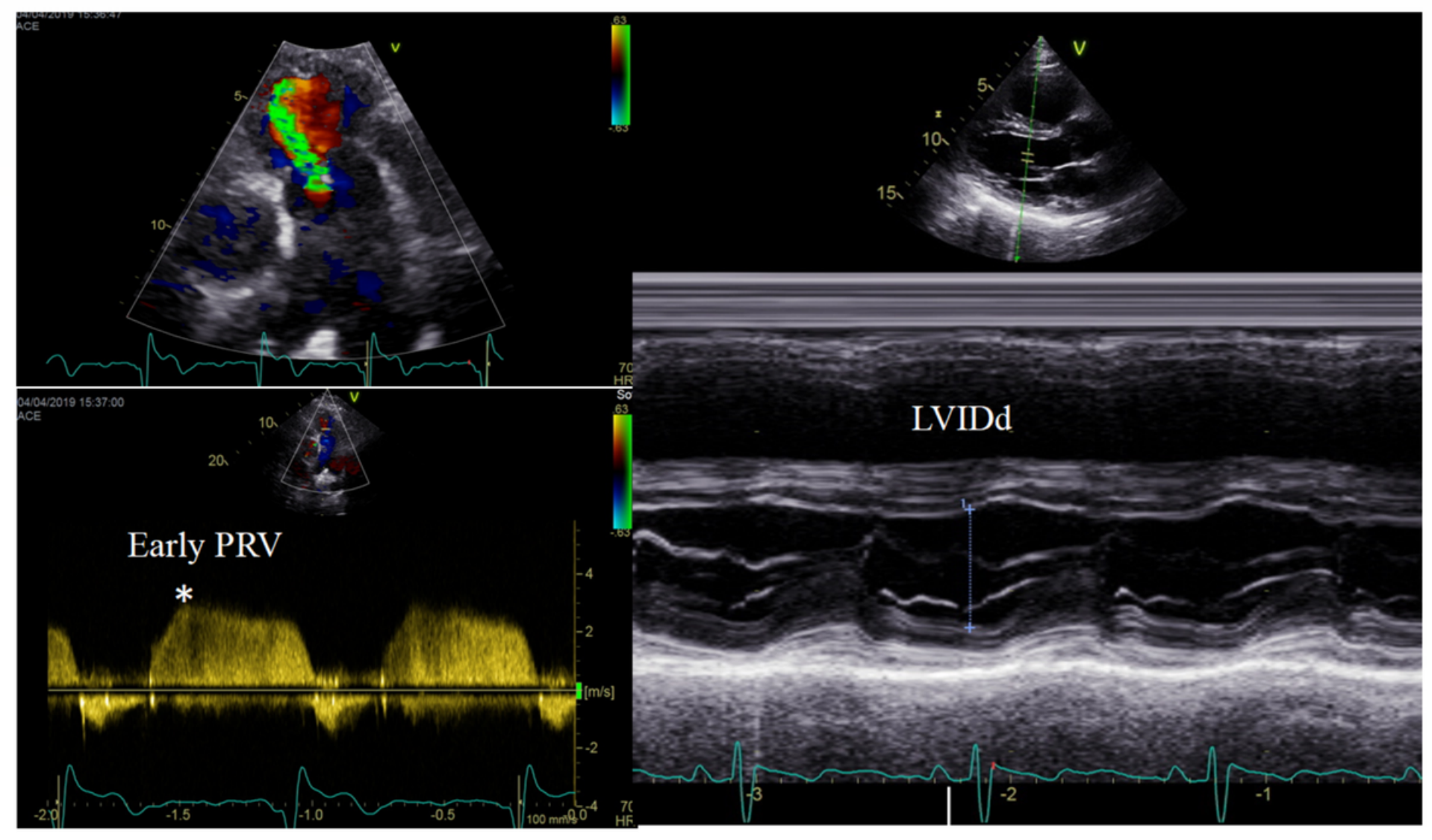

\section{Figure 2}

A. The early diastolic pulmonary regurgitation velocity (PRV) was measured to calculate mean pulmonary artery pressure. B. LV internal diameter at end-diastole (LVIDd) was acquired in the parasternal long-axis view at a level of the mitral leaflet tips by M-mode. 
A

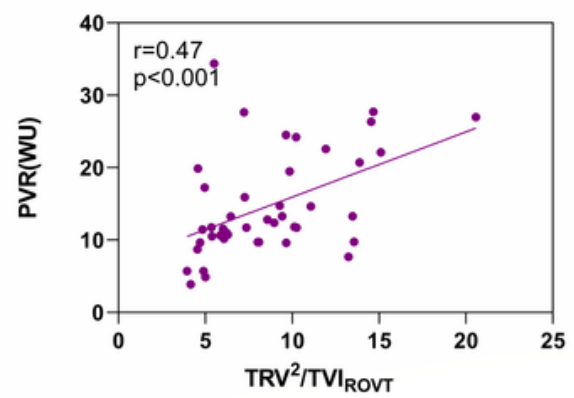

All patients

B

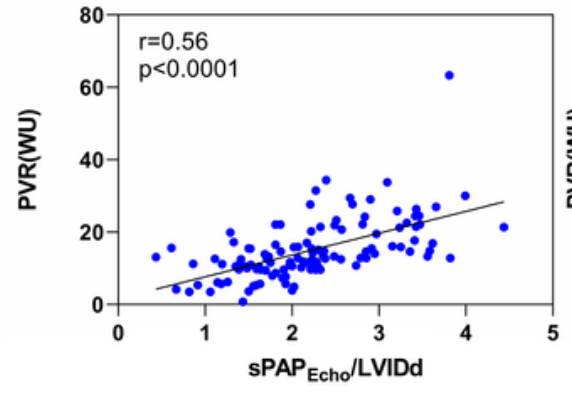

C

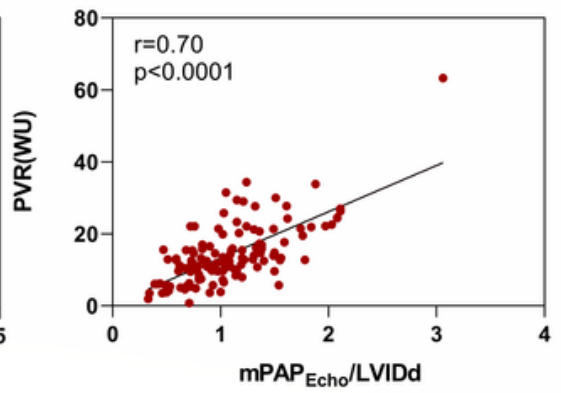

\section{Patients with PVR $\leqslant 15$ WU}

D

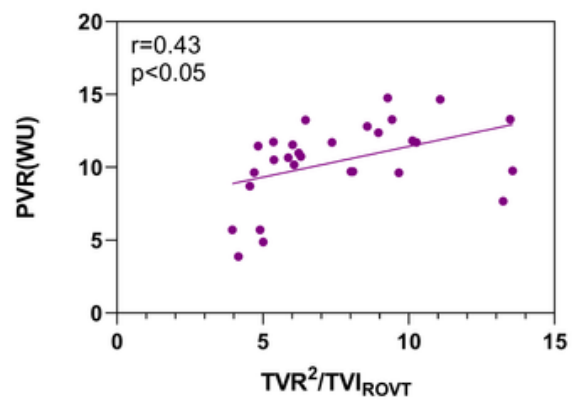

E

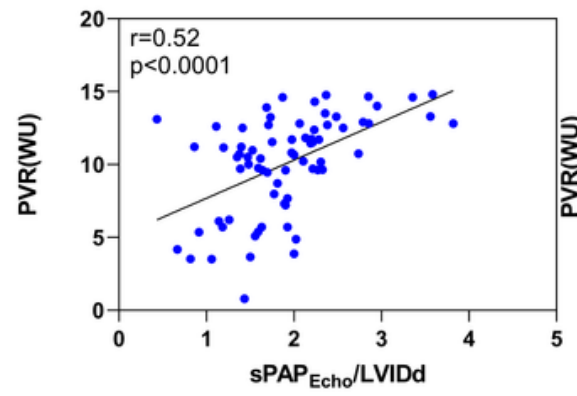

$\mathbf{F}$

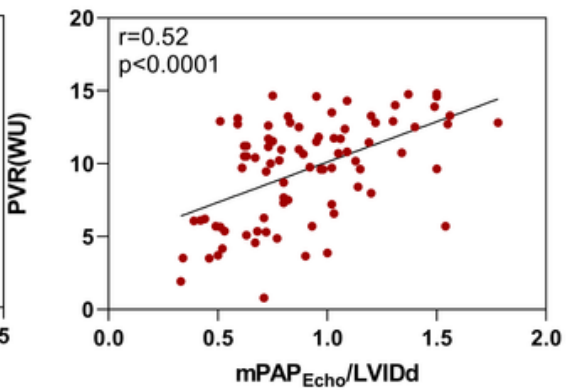

\section{Patients with PVR > 15 WU}

G

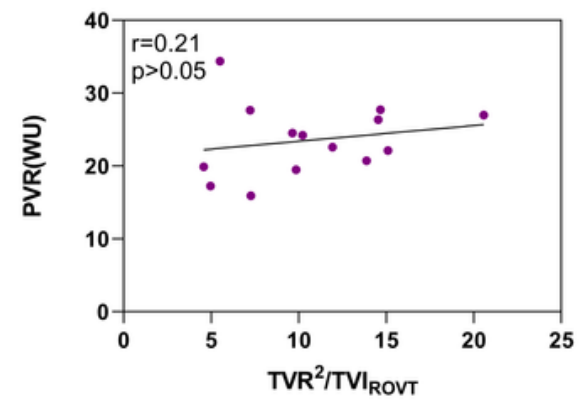

$\mathrm{H}$

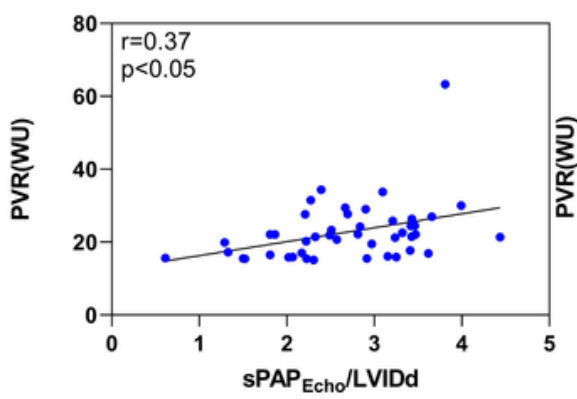

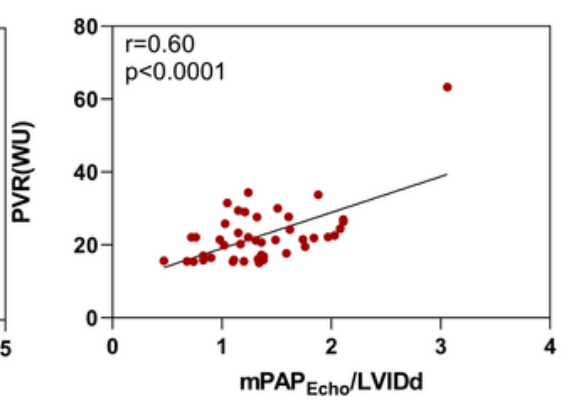

Figure 3

Linear regression analysis between PVR and TRV2 /TVIRVOT , sPAPEcho / LVIDd and mPAPEcho / LVIDd in the entire study cohort $(A \sim C)$, in patients with PVR $\leq 15 \mathrm{WU}(D \sim F)$, and in patients with $P V R>15 \mathrm{WU}$ (G I). 


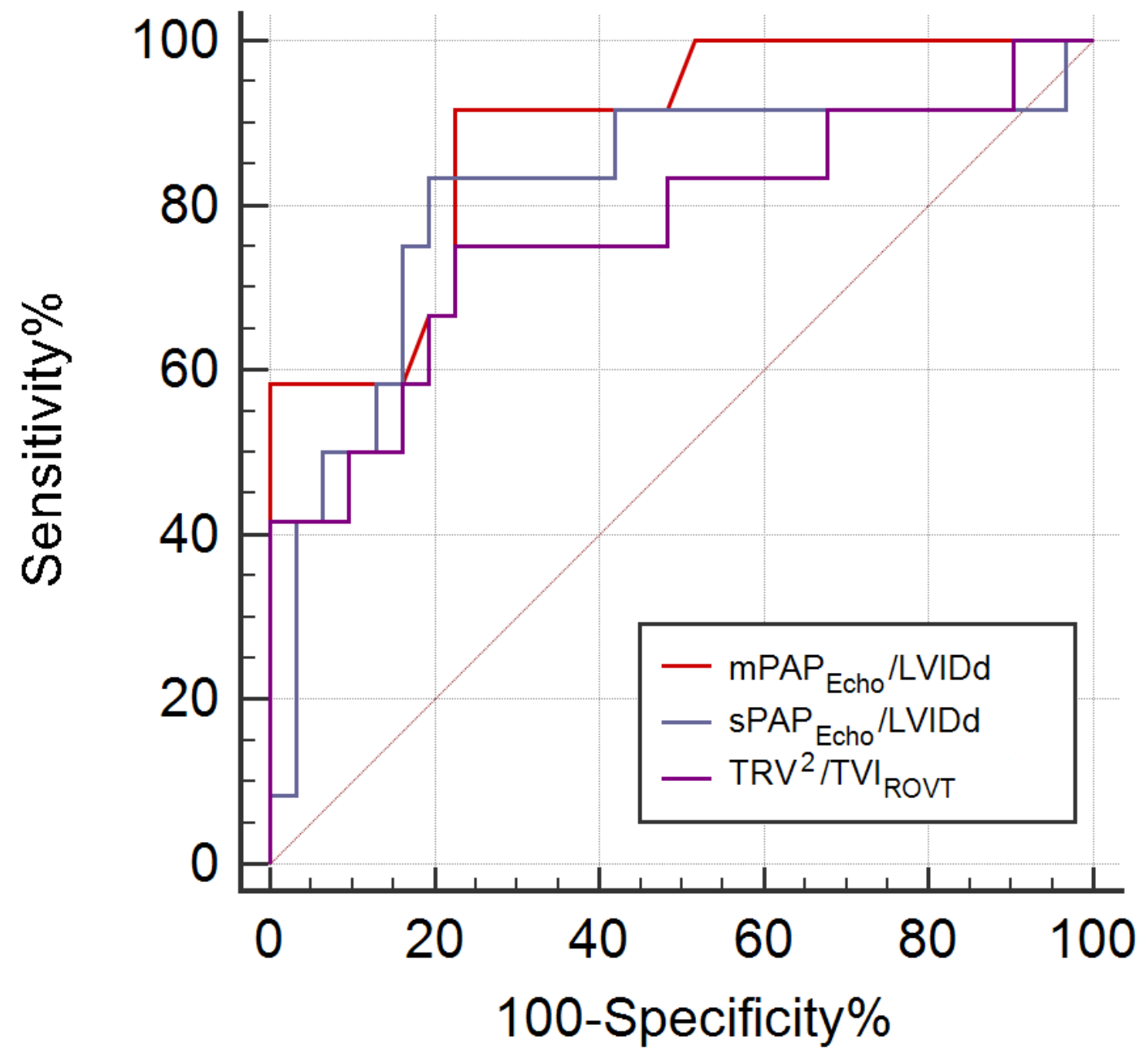

Figure 4

Receiver operating characteristic curves for TRV2 /TVIRVOT, sPAPEcho / LVIDd and mPAPEcho / LVIDd to distinguish PVR $>15$ WU. 